\title{
Modeling propagation in large deformed step index fibers using a finite operator method
}

\author{
Das S. Kumar ${ }^{1, *}$, Stephen C. Creagh ${ }^{2}$, Slawomir Sujecki ${ }^{1}$, and Trevor M. Benson ${ }^{1}$ \\ ${ }^{1}$ George Green Institute for Electromagnetics Research, University of Nottingham, University Park, Nottingham NG7 2RD, UK \\ ${ }^{2}$ School of Mathematics, University of Nottingham, University Park, Nottingham NG7 2RD, UK \\ *Corresponding author: sandip2mail@gmail.com
}

Compiled April 27, 2019

\begin{abstract}
A finite operator model is applied to the propagation of light in a deformed step-index fibers. The distribution of the light captured by the fiber from an arbitrary initial excitation is illustrated in the phase space for each fiber boundary. The method proves to be promising in modeling the transmission of light in the presence of fiber asymmetries. Simulations are made of the captured power in the core in the presence of fiber deformations. () 2019 Optical Society of America
\end{abstract}

OCIS codes: (80.5084) Phase space methods of analysis; (060.2310) Fiber optics.

http://dx.doi.org/10.1364/ao.XX.XXXXXX

\section{INTRODUCTION}

A finite operator model is developed in this paper to study the propagation of light in deformed, i.e. non-circular but longitudinally invariant, optical fibers. The emergence of mid-infrared (MIR) photonics using MIR-transparent chalcogenide glasses [1] motivates the investigations reported in this paper. This has included the fabrication of mid-infrared (MIR) fluorescence sources based on rare-earth (RE) -ion-doped chalcogenide glass optical fibers for MIR remote sensing applications $[2,3]$ and the development of advanced purification processes to reduce propagation losses in chalcogenide glass optical fibers [4,5]. We note, in particular, recent reports of luminescence from rare-earth-iondoped unstructured (i.e. single index) fibers whose diameters were of the order of $350 \mu \mathrm{m}[6,7]$ and optical transmission measurements on unstructured fibers of diameter $\sim 200 \mu \mathrm{m}$ across the wavelength range from 2-10 $\mu \mathrm{m}[4,8]$.

The drawing of a core-clad chalcogenide glass optical fiber from an extruded preform was reported in [9] where the core diameter is calculated as $148 \pm 4 \mu \mathrm{m}$ in a $222 \mu \mathrm{m}$ diameter cross section of fiber. As noted in [10] compared to unclad and largecore step-index RE-ion doped chalcogenide glass fiber, there is much less work in the literature, on small-core step-index, REion doped chalcogenide-glass fiber. A subsequent rod-in-tube step has been used in our group to fabricate small core small core step index fiber $[10,11]$, but it is challenging to obtain a step index fiber with a circular core using this method. The contrast of the viscosities of the two core/cladding glasses and the difficulties of aligning a core glass cane at the precise center of the cladding tube prior to fiber drawing the rod-in-tube preform are contributing factors [11]. Experimental analysis done on erbium doped fiber amplifiers (EDFA) by Doya et al
[12-14] suggests that pump light is absorbed more effectively in deformed optical fibers than in symmetric ones owing to the chaotic rays uniformly distributing the pump power inside the fiber. This motivated the study reported in the present paper of propagation in unstructured and step-index chalcogenide glass fibers possessing the noncircular but longitudinally uniform deformations observed in some experimental chalcogenide glass fibers. Investigation of deformed optical waveguides invokes the analysis of quantum and wave chaos systems [15? ,16], and involves investigation of chaotic light in the geometrical limit of ray-billiard systems. In asymmetric optical and microwave waveguides, the interference of chaotic rays scattered due to boundary deformations has been investigated extensively for asymmetric dielectric resonant cavities for application in directional emitters [17-19], and in active optical fibers [20-22] for selectively amplifying the modes in scars.

Light propagation in optical fibers is tracked using Poincaré surfaces-of-section (SOS) [23] which are constructed for each of the core and cladding boundaries of the SIF: individual rays are tracked using coordinates that record the position and orientation with which the ray intersects one of these boundaries and the corresponding phase planes then define the SOSs. Simple ray-tracing in the SIF becomes intractable very quickly because the power incident on the core-cladding dielectric boundary is split between reflected and transmitted rays. As a result, the total number of rays required to be traced over successive reflections increases exponentially. In order to overcome this drawback, the SOSs are coarse-grained, and a probabilistic approach is adopted in the measurement of the dynamics.

The discretization of the SOSs allows propagation of initial densities by a finite-dimensional matrix, formed by computing 
mapping probabilities for rays hopping from one region of a SOS to another. This finite matrix is a discrete representation of the Perron-Frobenius operator (PFO) for the problem [24]. Coarse-grained or discretized representations of PFOs have been extensively investigated in the past (see, for example, [25-27]). Of particular relevance to this paper is the simulation using similarly pixelated PFOs of the fully-chaotic SOS of deformed micro-resonant optical cavities in [28, 29]. The calculations in this paper are distinct in allowing coupling between multiple domains (core and cladding) and explicitly accounting for the third dimension.

Owing to the discretization used, there is a finite resolution with which the mixed SOS features can be resolved. The finite operator approximates these features and creates non-physical diffusive artefacts in the dynamics, which limits the number of reflections that can be performed. However, we will show that in spite of these limitations, the method proposed can be effectively employed to measure the net power accumulation in the core in deformed SIFs, from arbitrary input distributions. The formal basis for this analysis is discussed in section 2 . The discretization procedure and the corresponding propagation of pixelated densities is discussed in section 3, primarily in the context of a $2 \mathrm{D}$ projections of the dynamics. In section 4 we set out the core method proposed to simulate, in a fully 3D setting, the net accumulation of power transfer to the core. The method is first illustrated by application to simpler diffusionfree dynamical systems, before applying it to a more general, arbitrarily deformed, SIF. Finally, we describe how the method is concretely applied to a range of input power distributions in section 5 .

\section{PRELIMINARIES}

In this section, we establish the underlying principles and notation that will later be used to propagate light in deformed optical fibers by the finite operator method. For most of the discussion in this section, it suffices to consider ray dynamics on the single boundary of an unstructured fiber (see also [28]), but for the more detailed applications in later sections we will treat step-index fibers (SIFs) and here define our notation accordingly.

\section{A. Notation for momentum coordinates}

In the case of a SIF it will be important to distinguish between the unit vector $\mathbf{p}$ defining a ray's direction, which suffices to describe ray dynamics where the refractive index is always the same, and the momentum vector, defined here as

$$
\mathbf{P}=n \mathbf{p},
$$

where $n$ is the local refractive index. We will treat ray dynamics in a SOS setting, recording their position and direction when they hit fiber or core boundaries. For this purpose in particular we use

$$
P_{z}=\hat{z} \cdot \mathbf{P}=n \cos \theta
$$

to denote the momentum component along the fiber axis and

$$
P=\hat{t} \cdot \mathbf{P}=n \sin \theta \sin \chi
$$

to denote the momentum component along the cross-sectional boundary (of the cladding or a core), where the angles $(\theta, \chi)$ and unit vectors $\hat{z}$ and $\hat{t}$ are defined in Fig. 1 .

When a ray reflects specularly from a boundary or is transmitted to another medium by Snell's law, the dynamics can be stated simply in this notation as

$$
\left(P_{z}\right)_{\text {in }}=\left(P_{z}\right)_{\text {out }} \text { and }(P)_{\text {in }}=(P)_{\text {out }} .
$$

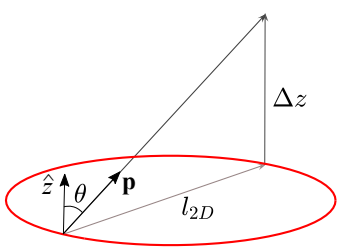

(a)

(b)

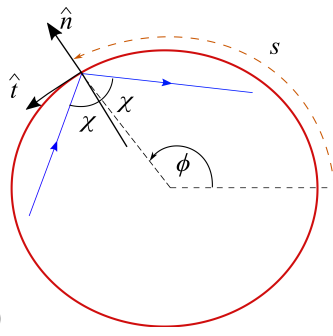

Fig. 1. (a) A ray with momentum vector $\mathbf{P}=n \mathbf{p}$, traverses a cross-sectional distance of $l_{2 D}$, and an axial distance of $\Delta z$. The vector $\mathbf{p}$ makes an angle of $\theta$ with the $z$-axis. (b) The ray is tracked in the SOS by recording the arc-length $s$, and the momentum $P$. Unit vectors normal and tangential to the boundary are given by $\hat{n}$, and $\hat{t}$ respectively.

In particular, $P_{z}$ is a global constant of motion, but note that the corresponding component $p_{z}$ of the unit vector $\mathbf{p}$ is not, in the case of a SIF. Note also that the momentum component $P$ used by us to parameterize cross-sectional ray direction differs in two respects from the typical parameterization of ray direction in $2 \mathrm{D}$ cavities by the simpler quantity $\sin \chi$ [28]. It includes the refractive index $n$, which of course changes between core and cladding, but also the factor

$$
\sin \theta=\sqrt{1-p_{z}^{2}}
$$

which also changes value on transmission from one medium to another. Use of this more complicated definition will simplify our comparison of phase-space densities of power at interfaces between different media and in the 3D context.

\section{B. Surface-of-section dynamics in 3D}

We record ray trajectories at each intersection with a boundary using SOS coordinates $\left(s, P, z, P_{z}\right)$, where $s$ denotes an arc-length coordinate along a cross-sectional boundary. For a particular trajectory, we use $\left(s_{m}, P_{m}, z_{m}, P_{z}\right)$ with $m=0,1,2 \cdots$ to record successive intersections with fiber boundaries, where $m=0$ labels the first such intersection following injection from the cross-sectional face of the fiber (recall that $P_{z}$ is constant). The dynamics

$$
(s, P) \rightarrow \varphi(s, P)
$$

of the cross-sectional components can be stated largely independently of the longitudinal coordinates $\left(z, P_{z}\right)$ (Although $P_{z}$ affects refraction angles in Snell's law, it is conserved and will therefore be suppressed in our notation later.) The longitudinal dynamics are then trivially determined using

$$
\left(z, P_{z}\right) \rightarrow\left(z+\Delta z\left(s, P, P_{z}\right), P_{z}\right),
$$

where

$$
\Delta z\left(s, P, P_{z}\right)=\frac{p_{z}}{\sqrt{1-p_{z}^{2}}} l_{2 D}(s, P)
$$

and $l_{2 D}(s, p)$ denotes the chord-length between intersections of the projection of the ray onto a $2 \mathrm{D}$ cross-section - as illustrated in Fig. 1.

\section{Ray densities on a 2D surface-of-section}

The full 3D ray dynamics of the optical fiber can be reduced to the analysis of light using 2D SOS, as will now be described. Let 
a power distribution of a beam of light arriving at the curved boundary be denoted by

$$
f\left(s, P, z, P_{z}\right)
$$

(where $f=0$ for $z<0$ ). Note that each point $\left(s, P, z, P_{z}\right)$ on a $4 \mathrm{D}$ SOS can be associated uniquely with a ray injected into the fiber's cross-sectional input face and arriving there after $m$ subsequent reflections/transmissions (or "bounces"). This allows us to partition the density along the entire extent of the boundary according to

$$
f\left(s, P, z, P_{z}\right)=\sum_{m=0}^{\infty} f_{m}\left(s, P, z, P_{z}\right),
$$

where $f_{m}\left(s, P, z, P_{z}\right)$ is the density restricted to rays with $m$ bounces. Note that we have defined canonical coordinates $\left(s, P, z, P_{z}\right)$ so that the corresponding net power flux is

$$
\Phi_{m}=\int_{\mathrm{SOS}} f_{m}\left(s, P, z, P_{z}\right) \mathrm{d} s \mathrm{~d} z \mathrm{~d} P \mathrm{~d} P_{z},
$$

which is conserved between bounces $\left(\Phi_{m+1}=\Phi_{m}\right)$ if the fiber is lossless.

Successive $m$-bounce densities are related by

$$
f_{m+1}\left(s, P, z, P_{z}\right)=f_{m}\left(\varphi^{-1}(s, P), z-\Delta z\left(s, P, P_{z}\right), P_{z}\right) .
$$

Since the $P_{z}$ coordinate is conserved between bounces, we will henceforth suppress it in our notation. We can then write (6) in the form

$$
f_{m+1}(s, P, z)=T_{3 D} f_{m}(s, P, z),
$$

where $T_{3 D}$ is the PFO, acting on an arbitrary function $g(s, P, z)$ by

$$
T_{3 D} g(s, P, z)=g\left(\varphi^{-1}(s, P), z-\Delta z(s, P)\right) .
$$

Although conservation of $P_{z}$ has allowed us to reduce the problem to iterating a function of the three variables $(s, P, z)$ (and to suppress $P_{z}$ in our notation), the relative simplicity of (3) allows us to reduce the dimension of the problem still further.

Denote the partial Laplace transform of $f_{m}(s, P, z)$ by

$$
\bar{f}_{m}(s, P, \alpha)=\mathcal{L}\left\{f_{m}(s, P, z)\right\}=\int_{z=0}^{\infty} \mathrm{e}^{-\alpha z} f_{m}(s, P, z) \mathrm{d} z
$$

and let

$$
\mathcal{L}\left\{T_{3 D} f_{m}(s, P, z)\right\}=\bar{T}_{3 D} \bar{f}_{m}(s, P, \alpha)
$$

define $\bar{T}_{3 \mathrm{D}}$. We use a Laplace representation because this provides a natural setting in which to treat initial-value problems (in which an input distribution is specified at $z=0$ ) and because it facilitates subsequent extension to problems with gain. Using the shifting theorem and (3), we may write

$$
\bar{T}_{3 D}=\mathrm{e}^{-\alpha \Delta z(s, P)} \bar{T}_{2 D},
$$

where

$$
\bar{T}_{2 D} h(s, P)=h\left(\varphi^{-1}(s, P)\right)
$$

defines $\bar{T}_{2 \mathrm{D}}$. Note that $\bar{T}_{2 D}$ is independent of the Laplace variable $\alpha$, and it operates only on a $2 \mathrm{D}$ projection of the full raydynamical system.

The problem has therefore effectively been reduced to a $2 \mathrm{D}$ one. There is still some dependence on the remaining coordinates because, in a SIF, refraction angles, reflectances and transmittances at material boundaries depend on the ignored constant $P_{z}$, but this reduction nevertheless represents an enormous savings when discretization of the operator is to be implemented in practice, as discussed in the next section. In principle, one must repeat the resulting linear algebra problems for each $\alpha$ to recover the full distributions in $z$, but note that the behavior of $\bar{f}_{m}$ for $\alpha \rightarrow 0$ suffices to characterize the solution in the limit $z \rightarrow \infty$, which is the relevant regime in applications. The resulting method is a more efficient alternative to brute force raytracing because the formation of the discretized finite operator only requires ray-tracing for one reflection. Once the mapping of all possible trajectories is done in the SOS for one reflection, $\bar{T}_{3 D}$ can be applied to a light distribution iteratively to obtain the distribution for the subsequent reflections.

\section{COARSE-GRAINING OF THE 2D PROJECTION}

In this section we describe the procedure used to form finite operators that approximate the transfer operators defined in Sec. 2 on a coarse-grained SOS. This coarse-graining is achieved by pixelating phase space and realizing the transfer operator as matrix of transition probabilities between pixels. The basic procedure is described in subsection $3 \mathrm{~A}$ and $3 \mathrm{~B}$. We assume a cladding with a 2D cross-sectional boundary described in polar coordinates $(r, \phi)$ by

$$
r=1+\epsilon \cos 2 \phi
$$

to exemplify the calculations, but emphasise that the method applies to more general shapes. In the remaining subsections we describe the extension of these calculations to SIFs and to the calculation of power transfer between core and cladding.

\section{A. Formation of a 2D finite operator}

To illustrate the coarse-graining of transfer operators we first consider the case of an unstructured fiber whose 2D cross-sectional SOS consists of a single component with coordinates $(s, p)$, where $0<s<s_{\max }$ and $s_{\max }$ denotes the circumference of the boundary. In a single 2D unstructured fiber, we may work with the simple direction cosine $p$ rather than the variable $P=n p$, and this lies in the range $-1<p<1$. This SOS is divided into $N \times N$ pixels, the case with $N=5$ being illustrated in Fig. 2(a). One of the pixels in Fig. 2(a) is filled with an ensemble of ray coordinates, whose mapping after one bounce is shown in Fig. 2(b). This concrete example corresponds to a fiber with boundary described by (13) with $\epsilon=0.11$. Counting the number of rays in each pixel after reflection allows us to approximate the transition probabilities between initial and final pixels, and thus to define the discretized transfer matrix as formalized below.
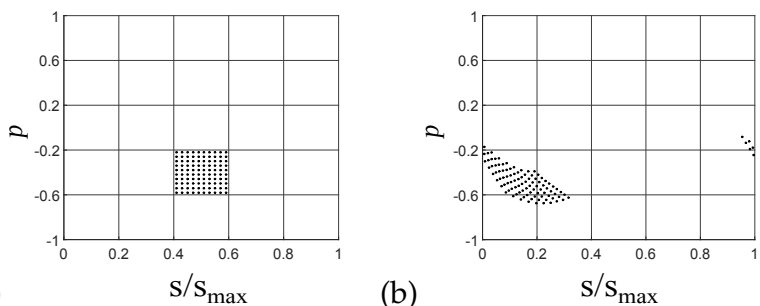

Fig. 2. (a) A SOS is divided into $N \times N$ pixels $(N=5)$, and an ensemble of rays uniformly distributed over a pixel is traced. (b) The probability of mapping from one pixel to next is calculated by tracing the ray ensemble for one bounce.

The fraction of power from the $j^{\text {th }}$ pixel moving to the $i^{\text {th }}$ pixel is denoted $t_{i j}$. These mapping fractions are then arranged 
to form the columns of a matrix $T_{2 D}$, such that

$$
T_{2 D}=\left(\begin{array}{ccccc}
\cdot & \cdots & t_{1 j} & \cdots & . \\
\vdots & \cdots & \vdots & \cdots & \vdots \\
t_{i 1} & \cdots & t_{i j} & \cdots & t_{i N^{2}} \\
\vdots & \cdots & \vdots & \cdots & \vdots \\
\cdot & \cdots & t_{N^{2} j} & \cdots & \cdot
\end{array}\right)_{N^{2} \times N^{2}}
$$

After the formation of $T_{2 D}$, any arbitrary distribution in the pixelated phase space can be propagated for any number of reflections by applying the matrix, $T_{2 \mathrm{D}}$, iteratively on the distribution. Raising $T_{2 \mathrm{D}}$ to the $m^{\text {th }}$ power and applying it to an initial distribution yields the distribution in the phase space after $m$ reflections, and is represented as

$$
\bar{f}_{m}=T_{2 \mathrm{D}}^{m} \cdot \bar{f}_{0}
$$

This provides the iterated ray distribution without explicitly using ray-tracing beyond the first reflection.

We provide in Appendix I a concrete illustration of the application of this discretization procedure in the context of a (2D projection of) an unstructured fiber. In particular, that illustration shows the origin of numerical diffusion that is an inherent part of pixelated propagation. A procedure is proposed in Sec. 4 to calculate transfer of power while mitigating such contamination but first, in the remainder of this section, we describe how pixelation is implemented in the context of SIFs.

\section{B. Extending the 2D finite operator for step index fibers}

We now describe an extension of the transfer operator to the case of a SIF with multiple boundaries between dielectric media - in fact, we restrict our detailed discussion to the case of a single core, but the basic discussion extends to multiple cores. To achieve this we construct a total phase space consisting of three separate phase planes (or SOS components): one denoted SOS(i) for rays on the core side of the core-cladding boundary; one denoted SOS(e) for rays on the cladding side of the corecladding boundary and one denoted SOS(c) for rays hitting the cladding boundary (which we assume to be totally reflecting). These are illustrated schematically in figure 3.

The cladding SOS (c), core exterior boundary SOS (e), and the core interior SOS (i) are respectively divided into $N_{\mathrm{C}} \times N_{\mathrm{c}}$, $N_{\mathrm{e}} \times N_{\mathrm{e}}$, and $N_{\mathrm{i}} \times N_{\mathrm{i}}$ pixels, and the resulting matrix, $T_{2 \mathrm{D}}$, now consists of $3 \times 3$ sub-block matrices denoted as

$$
T_{2 \mathrm{D}}=\left[\begin{array}{ccc}
T_{\mathrm{cc}} & T_{\mathrm{ce}} & T_{\mathrm{ci}} \\
T_{\mathrm{ec}} & T_{\mathrm{ee}} & T_{\mathrm{ei}} \\
T_{\mathrm{ic}} & T_{\mathrm{ie}} & T_{\mathrm{ii}}
\end{array}\right],
$$

where subscripts c, e, and i refer to the cladding, core exterior, and core interior SOSs respectively. For simplicity, all three dimensions are chosen to be equal in the explicit calculations performed here, so that $N_{\mathrm{c}}=N_{\mathrm{e}}=N_{\mathrm{i}}=N$.

Furthermore, the light distribution is also distinguished between intensity arriving at the boundary, and intensity leaving the boundary. In this section we let distributions arriving at the boundary be labelled with a '-' superscript and distributions leaving the boundary be labelled with a ' + ' superscript. Let $\bar{T}_{\text {ref }}$ be a reflection and transmission function at the boundaries that scatters a light distribution $\bar{f}_{m}^{-}$, incident on the boundaries

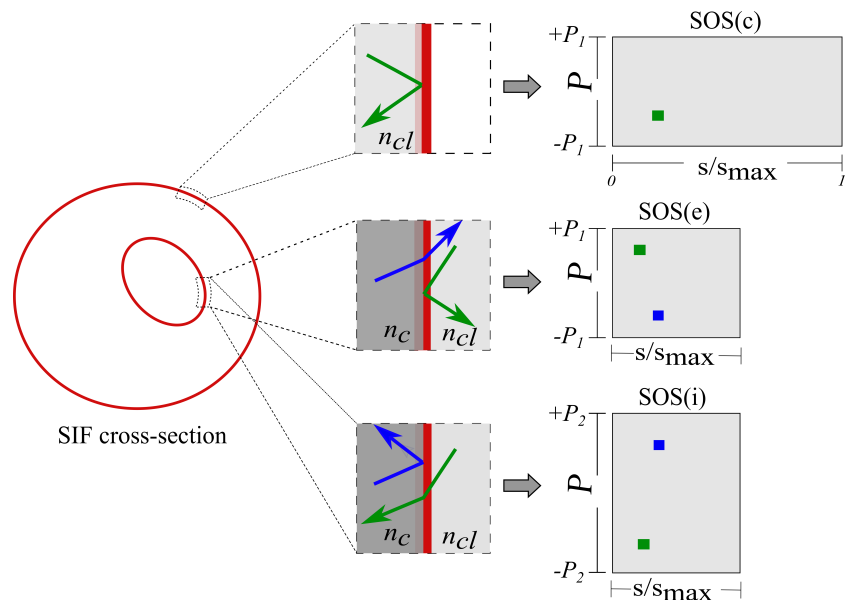

Fig. 3. Formation of the separate SOSs for propagating rays in the SIF are illustrated. The coordinate $P$ lies in the range $-P_{1}<P<P_{1}$, where $P_{1}=\sqrt{ }\left(n_{\mathrm{cl}}^{2}-P_{z}^{2}\right)$, in the cladding medium and in the range $-P_{2}<P<P_{2}$, where $P_{2}=\sqrt{ }\left(n_{\mathrm{c}}^{2}-P_{z}^{2}\right)$, in the core medium. The horizontal axes are scaled in each case by the circumference $s_{\max }$ of the cladding boundary.

after $m$ previous bounces, into a distribution $\bar{f}_{m}^{+}$, leaving the boundaries, such that

$$
\bar{f}_{m}^{+}=\bar{T}_{\text {ref }} \bar{f}_{m}^{-} .
$$

The matrix $\bar{T}_{\text {ref }}$ has block form

$$
\bar{T}_{\text {ref }}=\left[\begin{array}{ccc}
I & 0 & 0 \\
0 & \mathcal{R}_{\text {ext }} & \mathcal{T}_{\text {int }} \\
0 & \mathcal{T}_{\text {ext }} & \mathcal{R}_{\text {int }}
\end{array}\right],
$$

where $I$ is an identity matrix, $\mathcal{R}_{\text {ext }}$ and $\mathcal{T}_{\text {ext }}$ consist of reflectance and transmittance functions for power incident on the core exterior boundary and $\mathcal{R}_{\text {int }}$ and $\mathcal{T}_{\text {int }}$ consist of reflectance and transmittance functions for power incident on the core interior boundary.

The intensity distribution leaving each boundary is transported across the core or cladding interior until it is again incident on the boundaries, just before the next reflection or transmission. Let a transport operator $\bar{T}_{\text {int }}$ map the distribution just after the $m^{\text {th }}$ bounce into the distribution just before the $(m+1)^{\text {th }}$ bounce, so that

$$
\bar{f}_{m+1}^{-}=\bar{T}_{\text {int }} \bar{f}_{m}^{+} .
$$

The 2D finite operator used to iterate densities is then

$$
T_{2 \mathrm{D}}=\bar{T}_{\text {int }} \bar{T}_{\text {ref }}
$$

In the rest of this paper, we will adopt the convention that, where a superscript + or - is not used, the corresponding distribution is for power arriving at a boundary component.

A scalar approximation is adopted in order to find the division of power between the transmitted ray and the reflected ray at the core-cladding dielectric boundary. Fresnel's equation for TE polarization is used to find the reflectance and transmittance of the rays traveling in and out of the core boundary. The reflectance then

$$
\mathcal{R}=\left|\frac{A-B}{A+B}\right|^{2},
$$


where

$$
\begin{aligned}
A & =\sqrt{n_{\mathrm{inc}}^{2}-P^{2}-P_{z}^{2}} \\
B & =\sqrt{n_{\text {trans }}^{2}-P^{2}-P_{z}^{2}} .
\end{aligned}
$$

The corresponding transmittance is

$$
\mathcal{T}=1-\mathcal{R},
$$

where we uses lossless models of ray propagation and scattering.

\section{Two examples of SIF geometry}

We now illustrate the concrete application of the finite transfer operator to SIFs, concentrating on two examples. The first is that of a circular core inside a circular cladding. The phase space of this configuration has significant chaotic components if core and cladding are non-concentric, but the border between chaotic and regular is a simple horizontal line in the phase plane, which is aligned with the pixelation so that there is no diffusive leakage between them. This allows us later to test calculations of power transfer between core and cladding in a context in which diffusion artefacts do not play a significant role. The second example is of a deformed core and cladding, presenting a more generic scenario in which numerical diffusion between chaotic and regular regions dominates calculations of power transfer for large numbers of iterations.

The configuration of the circular core-cladding example is illustrated in Fig. 4(a). Here the outer cladding boundary is a unit circle, and the core is circular with radius $r=0.2828$, centered at $(0.1,0.3)$. The area of the core is $8 \%$ of the cross-sectional area of the cladding. The refractive index of the core is $n_{\mathrm{c}}=2.70$ and that of the cladding medium is $n_{\mathrm{cl}}=2.55$, typical of MIRtransparent chalcogenide glasses. We propagate an initial light distribution, with global constant $P_{z}=0.75 n_{\mathrm{cl}}$, which uniformly illuminates the cladding SOS and vanishes on the core interior and exterior SOSs: see the initial row of Fig. 4(b). We emphasise that this somewhat unphysical initial condition is chosen primarily to simplify the preliminary description of phase space evolution: more realistic initial conditions are treated in Sec. 5.

Subsequent rows of Fig. 4(b) show the distribution after 30, 100 and 450 bounces labeled 1, 2 and 3 respectively. The coreinterior and cladding SOSs each have invariant whisperinggallery regions of trapped light (by total internal reflection in the core and by orbits missing the core between total reflections in the cladding). Between them are chaotic regions, and the SOS corresponding to the core exterior is also totally chaotic. Light can neither enter nor leave these regions and the pixelation is such that the finite operator respects this invariance: because the pixels are aligned with the borders between chaotic and whispering-gallery regions, there can be diffusion along the border but not across it. The whispering gallery regions of the core interior, which satisfy $|P|>n_{\mathrm{cl}} / n_{\mathrm{c}}=2.55 / 2.70$, then remain un-illuminated indefinitely as the finite transfer operator is iterated. Likewise, the whispering-gallery bands of the cladding SOS remain uniformly illuminated as in the initial distribution. There is mixing, however, between these invariant regions and between the core exterior. The distribution in this chaotic mixing region is still quite complex in row 1 of Fig. 4(b) (30 iterations), but is approaching a steady state in row 2 (100 iterations) and has effectively reached steady state in row 3 (450 iterations), where the coarse-grained density is uniform on the scales used.

We perform analogous calculations for a deformed SIF, illustrated in Fig. 5(a). In this second example the core is elliptical

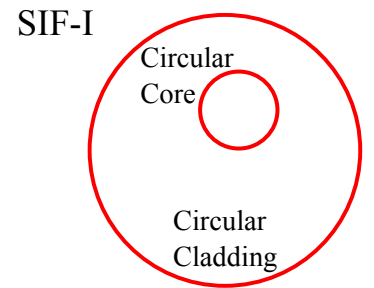

(a)
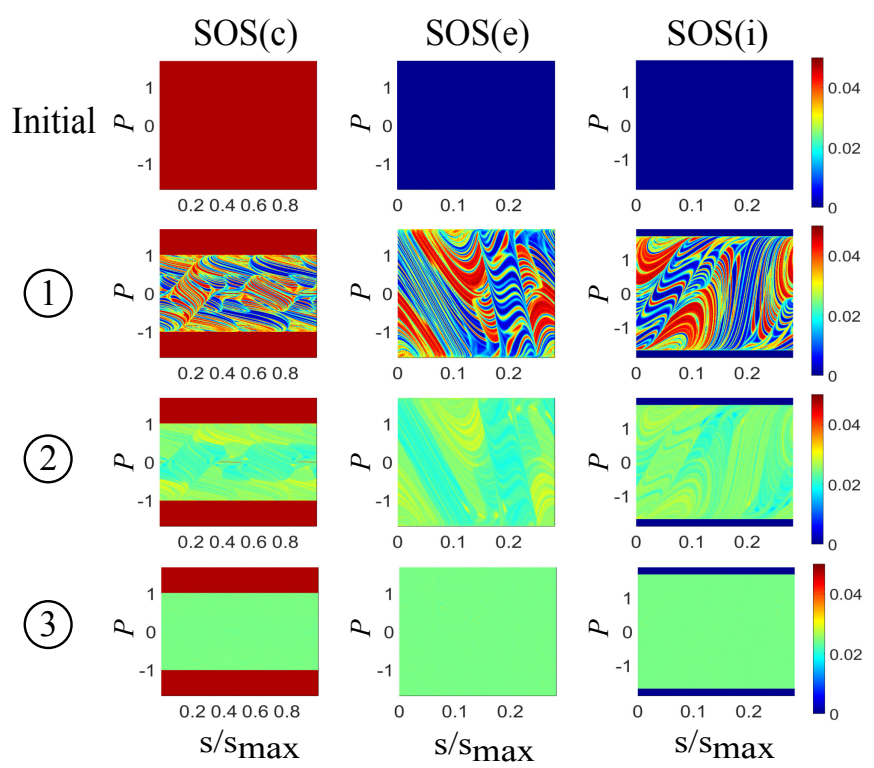

(b)

Fig. 4. (a) A SIF cross-section with circular core and cladding boundaries. (b) An initially uniform light distribution is excited from the cladding SOS and allowed to propagate in the fiber. SOS distributions are shown for the propagation after (1) $30^{\text {th }}$, (2) $100^{\text {th }}$, and (3) $450^{\text {th }}$ reflections.

and centered at $(0.1,0.3)$, with major axis 0.4 , minor axis 0.2 and with the major axis oriented at an angle of $135^{\circ}$ to the $x$ axis. The cladding boundary is also deformed, of the form (13) with $\epsilon=0.05$. This core has the same cross-sectional area as in the previous example and we also choose refractive indices to have the same values as used there.

As in the first example, there are invariant, regular whispering gallery regions in the cladding and core-interior SOSs, with mixing, and chaotic regions between them and through the core exterior SOS. The borders between regular and chaotic regions are no longer simple, however, and diffusion due to pixelation allows ray density to leak between them. This is evident in the evolution shown in Fig. 5(b). This follows iteration of the same initial distribution as used in the previous, circular example (uniform in the cladding SOS and vanishing on the core SOSs). As in Fig. 4(b), rows 1, 2 and 3 respectively show the distribution after 30, 100 and 450 bounces, but an additional row 4 with 1000 bounces is also shown as convergence to an effective steady state is slower in this case.

There are two significant differences to the previous example. First, and most importantly for later calculations, there is diffusion into and across whispering gallery regions. This is especially evident around the island chain in the region of the core interior SOS between whispering gallery and chaotic regions. 


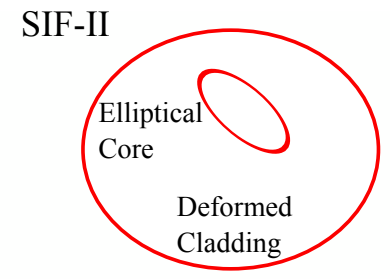

(a)
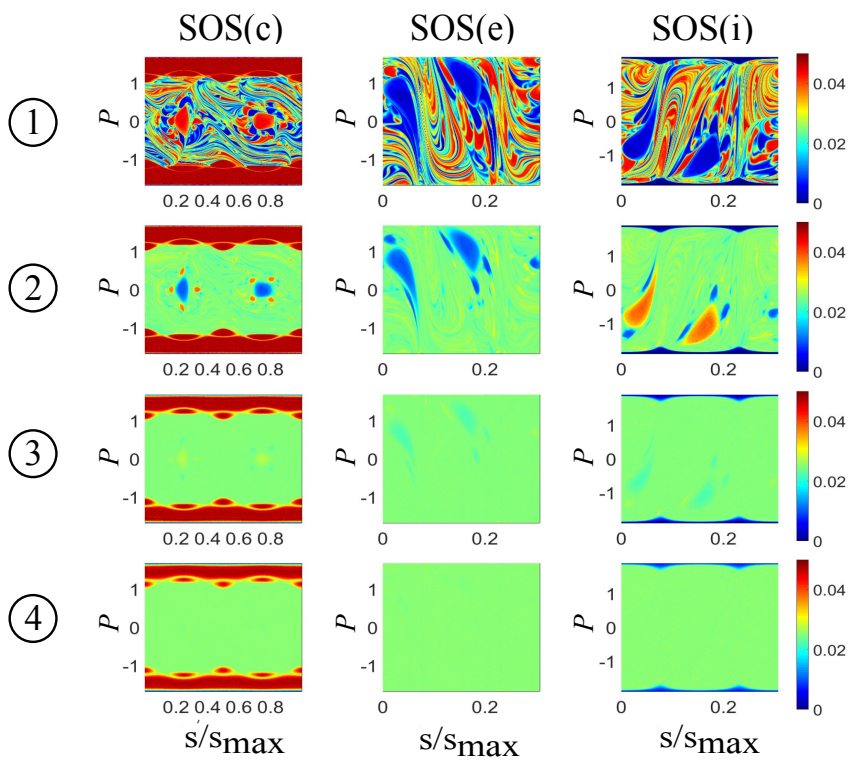

(b)

Fig. 5. (a) A deformed SIF cross-section. (b) the evolution in the fiber of a uniform distribution launched from the cladding SOS. The SOS distributions are shown after (1) $30^{\text {th }}$, (2) $100^{\text {th }}$, (3) $450^{\text {th }}$, and (4) $1000^{\text {th }}$ reflections.

One can see that the initially narrow separatrix band of chaos around the islands widens progressively with increasing iteration, which is unphysical. Such diffusive contamination of the trapped regions will eventually dominate and make simulations of flux transfer invalid unless we truncate iteration numbers. This aspect is pursued in the following section.

A less important, but nevertheless interesting, difference between this and the previous example is that there is a decaying island-like feature, prominent after 100 iterations in row 2, significantly reduced but still visible after 450 iterations, in row 3, and essentially gone after 1000 iterations, in row 4 . We term this a "leaky resonance" and its decay is physical, not a result of diffusion artefacts. It is associated with rays which refract through the core every time they hit it. The focusing effect of the core leads to a stable island structure which would remain invariant if there was total transmission at every bounce. However, although the transmittance is close to unity, there is some loss to reflection at each core-bounce and the island slowly decays with iteration number as a result. Numerical diffusion into the corresponding island is a much slower process than this (physical) decay. Its effect on calculations to follow will be to lead to longer, oscillating transients in the calculation of power transfer between cladding and core.

\section{Accumulation of power in the core in a 2D projection}

The finite operator method allows us to monitor flux transfer across the core boundary to capture the power carried by rays moving into and coming out of the core. The detailed 3D calculation is set out in Sec. $4 \mathrm{~A}$ but we first set up a simplified version of the power transfer problem applied to a $2 \mathrm{D}$ projection. This in particular establishes the limitations placed by numerical diffusion into and out of regions of phase space occupied by totally-internally reflected ray orbits (see Sec. 3C).

Power enters the core when a distribution incident on the core exterior boundary is transmitted through. Power leaves the core when a distribution incident on the core interior boundary is transmitted out. These combine to provide the net transfer of power into the core. We illustrate the procedure in this subsection by directly setting $\alpha=0$ in (11) so that we are effectively 2D setting in which

$$
\bar{T}_{3 \mathrm{D}} \rightarrow T_{2 \mathrm{D}}
$$

The net transfer of power from the cladding to the core at the $m^{\text {th }}$ bounce in this simplified 2D problem is then written as

$$
\Delta \Phi_{2 D}^{m}=\left\langle w, \bar{f}_{m}\right\rangle,
$$

where

$$
w(s, P)= \begin{cases}0 & \text { on } \operatorname{SOS}(\mathrm{c}) \\ \mathcal{T}_{\text {int }} & \text { on } \operatorname{SOS}(\mathrm{e}) \\ -\mathcal{T}_{\text {ext }} & \text { on SOS(i) }\end{cases}
$$

and $\bar{f}_{m}(s, P)$ is the density of rays arriving at the corresponding boundary.

The cumulative transfer of power, up to and including the $M^{\text {th }}$ reflection, is

$$
\begin{aligned}
\Phi_{2 \mathrm{D}}^{M} & =\sum_{m=0}^{M} \Delta \Phi_{2 D}^{m} \\
& =\sum_{m=0}^{M}\left\langle w, T_{2 D}^{m} \bar{f}_{0}\right\rangle .
\end{aligned}
$$

The quantity of interest in applications is the net transfer of power over the entire length of the fiber. The limit

$$
\Phi_{2 \mathrm{D}}=\lim _{M \rightarrow \infty} \sum_{m=0}^{M} \Phi_{2 D^{\prime}}^{M}
$$

is related to this quantity but is not the same, as will be discussed more fully in Sec. $4 \mathrm{~A}$, because it orders power transfer by the number of bounces rather than distance $z$ along the fiber. It is used in this section, however, to illustrate the limitations placed on simulations by diffusive effects.

We emphasize the approach to $\Phi_{2 D}$ as a limit in (28) for two reasons.

1 The limit exists even though its formal geometric sum

$$
\Phi_{2 \mathrm{D}}=\left\langle w,\left(I-T_{2 D}\right)^{-1} \bar{f}_{0}\right\rangle
$$

is ill-defined because the largest eigenvalue of $T_{2 D}$ is equal to 1 . The geometric sum can be regularized, as $w$ is necessarily orthogonal to the subspace corresponding to unit eigenvalues, but this procedure requires further discussion, as provided in the next section. 
2 More importantly from a practical point of view, unphysical diffusion artefacts may dominate the formal limit $M \rightarrow \infty$ when the core or cladding is deformed. We must in such applications therefore restrict $M$ to values for which numerical diffusion has a sufficiently small impact. Estimation of appropriate bounds for $M$ is the subject of the rest of this subsection.

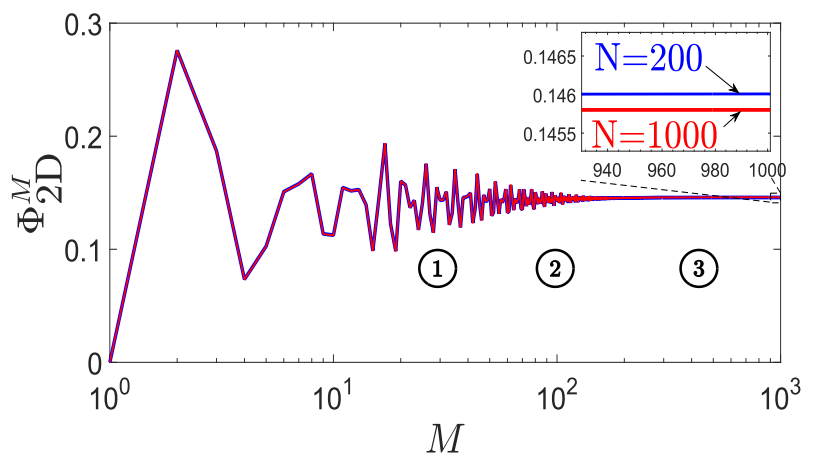

(a)

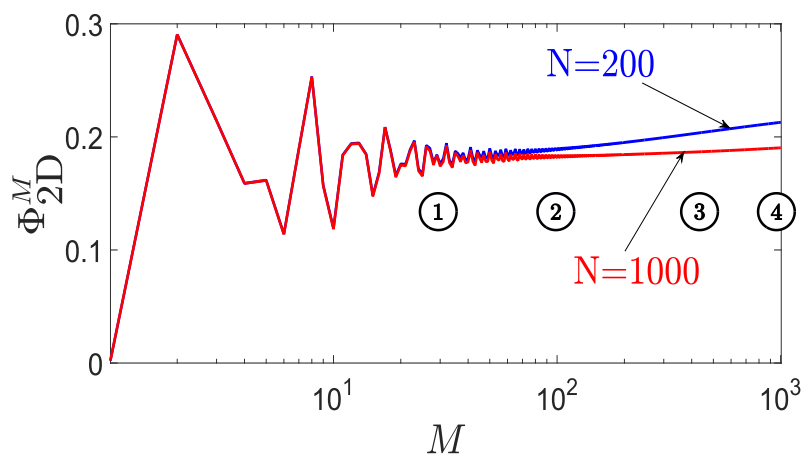

Fig. 6. (a) $\Phi_{2 D}^{M}$ calculated for SIF with circular core and cladding boundaries in figure 4. (b) $\Phi_{2 D}^{M}$ calculated for the deformed SIF in figure 5

We illustrate the effects of numerical diffusion using the two concrete examples described in Sec. 3C. The first example, with circular core and cladding (see Fig. 4) has insignificant diffusion and serves as a benchmark. The second example is the case of deformed core and cladding illustrated in Fig. 5 and is intended to be representative of generic problems where numerical diffusion plays a more prominent role. The evolution of $\Phi_{2 \mathrm{D}}^{M}$ with $M$ is shown in figure 6(a) for the benchmark, circular core-cladding problem. We see that $\Phi_{2 \mathrm{D}}^{M}$ has effectively converged to its asymptotic value $\Phi_{2 \mathrm{D}} \approx 0.146$ after the order of 100 reflections. Increasing pixel dimensions from $N=200$ to $N=1000$ leads to some convergence of this value towards a limit, but does not qualitatively change the picture.

In figure $6(\mathrm{~b})$ we show an equivalent calculation for the more generic example with deformed core and cladding. Here the border between regular whispering gallery regions and chaotic regions is no longer horizontal in phase space (see Fig. 5) so there is diffusive transport between the two. Unlike for the benchmark problem, $\Phi_{2 \mathrm{D}}^{M}$ does not reach a simple plateau here, but continues to drift upwards even after the initial transients have died out for $M$ between about 30 and about 100 . Furthermore, there is a marked difference between the calculations with $N=200$ and with $N=1000$, with the former showing significantly more drift.
This is because diffusion due to pixelation allows power to enter the invariant and totally internally reflected whispering gallery regions. This is an unphysical effect which can be postponed to higher reflection numbers, but not eliminated, by using finer pixelation. In practice therefore we must restrict our calculations to values of $M$ where these diffusion artefacts are not dominant. For the simulations illustrated in figure 6, calculations of flux become inaccurate once $M$ is larger than a few hundred, for example.

\section{EVOLUTION OF DENSITIES IN 3D GEOMETRIES}

In this section, we extend the discussion to treat the fully $3 \mathrm{D}$ evolution of power densities in SIFs, concentrating particularly on the calculation of asymptotic power in the core. We illustrate the calculation first with a toy model in Sec. 4B and then for the special case of non-concentric circular core and cladding geometries in Sec. $4 \mathrm{C}$ - both of these problems are not significantly limited by numerical diffusion, allowing us to illustrate underlying procedure in a simpler setting before application to more general problems in Sec. 4D.

\section{A. Power transfer in a 3D structure}

The net transfer of flux to the core over the fiber's entire length in a fully 3D representation of ray dynamics can be written in terms of the function $w$ defined in (26) as

$$
\Phi_{3 \mathrm{D}}=\int_{0}^{\infty}\langle w, f(z)\rangle \mathrm{d} z
$$

using the notation of Sec. $3 \mathrm{C}$ and where we suppress arguments $(s, P)$ in densities such as $f(s, P, z)$ in this section. We have proposed in Sec. 3D using a Laplace representation in which we replace

$$
f(z) \rightarrow \bar{f}(\alpha) .
$$

In this context we may formally write

$$
\Phi_{3 \mathrm{D}}=\lim _{\alpha \rightarrow 0}\langle w, \bar{f}(\alpha)\rangle .
$$

We have seen in Sec. 3D, however, that numerical diffusion arising from pixelation may require us in deformed geometries to constrain iterated densities to a finite number of bounces. In this context we must approach the limit $\alpha \rightarrow 0$ in the Laplace domain with care, as we now outline.

Let us define

$$
\bar{\Phi}_{3 \mathrm{D}}^{M}(\alpha)=\sum_{m=0}^{M}\left\langle w, \bar{f}_{m}(\alpha)\right\rangle,
$$

which calculates the net transfer of flux to the core, up to and including a fixed number $M$ of bounces. In problems without numerical diffusion, we evaluate (31) by letting both $M \rightarrow \infty$ and $\alpha \rightarrow 0$, however this must be done with care as the limits do not commute, as established in the next subsections. The required quantity (as defined by (31)) is

$$
\Phi_{3 \mathrm{D}}=\lim _{\alpha \rightarrow 0} \lim _{M \rightarrow \infty} \bar{\Phi}_{3 \mathrm{D}}^{M}(\alpha) .
$$

This is to be distinguished from the limit

$$
\Phi_{2 \mathrm{D}}=\lim _{M \rightarrow \infty} \lim _{\alpha \rightarrow 0} \bar{\Phi}_{3 \mathrm{D}}^{M}(\alpha),
$$

obtained by setting $\alpha$ to zero before iterating in bounce number, which is in fact the quantity previously calculated for a $2 \mathrm{D}$ 
projection of dynamics (and similarly denoted) in Sec. D (see (27)).

The relevance of

$$
\Phi_{3 \mathrm{D}} \neq \Phi_{2 \mathrm{D}}
$$

in practical terms is that, if we truncate $M$ to make allowance for diffusion artefacts, we must ensure that $\alpha$ remains sufficiently large that the truncation does not matter, but at the same time small enough to approximate (30) sufficiently well. Such a procedure is first applied to a toy model in Sec. 4B and then in Sec. $4 \mathrm{C}$ to a SIF with circular core and cladding boundaries. Both of these problems serve as benchmarks in which diffusion is not significant and in which we may compare our $M$-truncated calculation with the true limit $M \rightarrow \infty$. Finally, in Sec. $4 \mathrm{D}$, the calculation is applied to the deformed SIF where diffusion is present and truncation in $M$ is required.

\section{B. Power transfer in a toy model}

We first illustrate the relevance of the order of limits in (33), and the corresponding impact of truncating $M$, using a toy model for which the transfer operator is by definition a finite matrix and there is no diffusion mechanism.

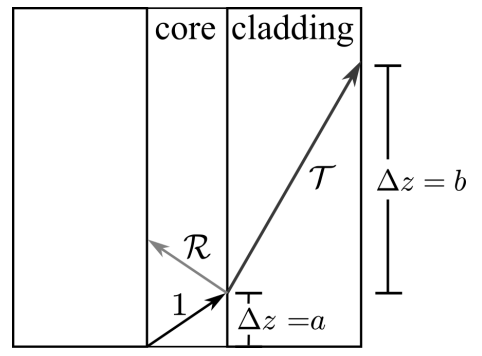

Fig. 7. A toy model consisting of a $2 \mathrm{D}$ waveguide for $z$ propagation along the fiber.

The model consists of a 2D waveguide, illustrated in Fig. 7. The SOSs here are zero-dimensional, so there are no $(s, P)$ coordinates and the entire boundary of core or cladding is described by canonical coordinates $\left(z, P_{z}\right)$. As usual, $P_{z}$ is a global constant of motion, and is suppressed in our notation. In fact, we restrict our attention to densities that are symmetric about the middle axis of the waveguide so that the cross-sectional SOSs each consist of a single point. The transfer matrix reduces to the $3 \times 3$ matrix

$$
\bar{T}_{3 \mathrm{D}}(\alpha) \rightarrow \bar{T}_{\text {toy }}(\alpha)=\left[\begin{array}{ccc}
0 & \mathcal{R} \mathrm{e}^{-\alpha b} & \mathcal{T} \mathrm{e}^{-\alpha b} \\
\mathrm{e}^{-\alpha b} & 0 & 0 \\
0 & \mathcal{T} \mathrm{e}^{-\alpha a} & \mathcal{R} \mathrm{e}^{-\alpha a}
\end{array}\right]
$$

Here, $\mathcal{T}$ and $\mathcal{R}$ are respectively the reflectance and transmittance of a ray hitting the core-cladding boundary and $\Delta z=a$ and $\Delta z=b$ are the vertical displacements experienced of a ray traversing the core or the region between core and cladding. All of these would depend on $P_{z}$, but we reiterate that $P_{z}$ is a constant of motion and so they are effectively fixed for the rest of this discussion.

The net transfer of flux to the core is once again represented by (30) except that $w$ is now the finite vector

$$
w=\left[\begin{array}{lll}
0 & \mathcal{T} & -\mathcal{T}
\end{array}\right]
$$

(We reuse symbols such as $\Phi_{3 \mathrm{D}}$ and $\Phi_{2 \mathrm{D}}$ here even though the underlying geometries are now respectively two-dimensional and one-dimensional.) Since there are no diffusion artefacts in this model, we may pass directly to the limit $M \rightarrow \infty$, allowing us to write, for example

$$
\Phi_{3 \mathrm{D}}=\lim _{\alpha \rightarrow 0}\left\langle w, \frac{1}{1-\bar{T}_{\text {toy }}(\alpha)} \bar{f}_{0}\right\rangle .
$$

The gap between $\Phi_{3 \mathrm{D}}$ and $\Phi_{2 \mathrm{D}}$ in (33) is directly explained in this context by representing the right-hand side of (36) using a basis of eigenvectors of $\bar{T}_{\text {toy }}(\alpha)$. The gap is in particular determined by the leading eigenvalue $\lambda_{0}(\alpha)$ which has the limit

$$
\lim _{\alpha \rightarrow 0} \lambda_{0}(\alpha)=1
$$

We note that $w$ is necessarily orthogonal to the corresponding eigenvector, which we denote as $u_{0}(\alpha)$, in this limit:

$$
\lim _{\alpha \rightarrow 0}\left\langle w, u_{0}(\alpha)\right\rangle=0
$$

Using l'Hôpital's rule, we therefore find that the contribution of this leading eigenvector to (36) is of the form

$$
\delta \Phi=\text { const. } \times \frac{\left\langle w, u_{0}^{\prime}(0)\right\rangle}{\lambda_{0}^{\prime}(0)},
$$

where the primes denote a derivative with respect to $\alpha$, evaluated at $\alpha=0$. An analogous evaluation of the truncated sum $\bar{\Phi}_{3 \mathrm{D}}^{M}(\alpha)$ has no contribution from this leading eigenvector in the limit $\alpha \rightarrow 0$, because of (38), while all other eigenvectors contribute identically in the limit $M \rightarrow \infty$. Therefore this leadingeigenvalue contribution is the origin of the gap

$$
\Phi_{3 \mathrm{D}}-\Phi_{2 \mathrm{D}}=\delta \Phi
$$

in (33) that arises from the exchange of limits $\alpha \rightarrow 0$ and $M \rightarrow \infty$.

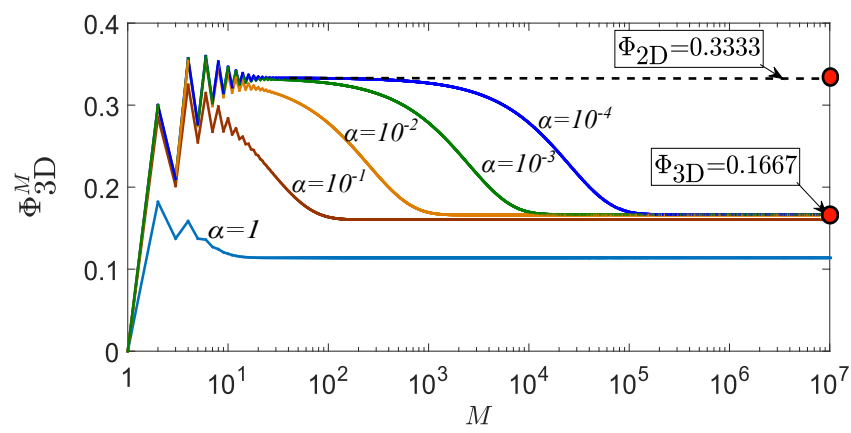

Fig. 8. The relevance of the order of limits $\alpha \rightarrow 0$ and $M \rightarrow \infty$ in $\bar{\Phi}_{3 \mathrm{D}}^{M}(\alpha)$ is illustrated for the toy model. For each $\alpha, \bar{\Phi}_{3 \mathrm{D}}^{M}(\alpha)$ approaches a plateau as $M \rightarrow \infty$, whose level in turn approaches $\Phi_{3 \mathrm{D}}$ as $\alpha \rightarrow 0$. However, for smaller values of $\alpha$, $\bar{\Phi}_{3 \mathrm{D}}^{M}(\alpha)$ first approaches a higher-value plateau for intermediate $M$, followed by a transition to the final plateau that occurs for larger values of $M$ as $\alpha$ decreases. As $\alpha \rightarrow 0$, the level of this intermediate plateau approaches $\Phi_{2 \mathrm{D}}$.

We now set out the ramifications of this gap for calculations of the net transfer of flux when $M$ is truncated. We perform concrete calculations for the special case $\bar{f}_{0}=[1,0,0]$, so that the initial distribution illuminates the cladding boundary only, and 
for $a=0.2, b=0.5, \mathcal{R}=0.7$ and $\mathcal{T}=0.3$. Figure 8 plots the resulting $\bar{\Phi}_{3 \mathrm{D}}^{M}$ as a function of $M$ for several values of $\alpha$. For each fixed $\alpha, \bar{\Phi}_{3 \mathrm{D}}^{M}$ approaches a plateau as $M \rightarrow \infty$. As $\alpha$ decreases these plateau values approach

$$
\Phi_{3 \mathrm{D}}=\frac{a}{a+2 b}=\frac{1}{6}
$$

which can be obtained directly from (36). On the other hand, if $M$ is capped at some possibly large but fixed value, as it must be in problems with diffusion, a different limit

$$
\Phi_{2 \mathrm{D}}=\frac{1}{3}
$$

indicated by a dashed line in Fig. 8, is obtained as $\alpha \rightarrow 0$. The physical result $\Phi_{3 \mathrm{D}}$ takes account of the extent of the core relative to the cladding, whereas $\Phi_{2 \mathrm{D}}$ provides a topological weighting by number of bounces of each of the SOSs and is independent of geometrical dimensions $a$ and $b$.

In this toy model it is unproblematic to take the limit $M \rightarrow \infty$, or indeed to pass directly the limiting value in (36), in order to find the geometrical result $\Phi_{3 \mathrm{D}}$. In problems limited by numerical diffusion, however, we must estimate $\Phi_{3 \mathrm{D}}$ while keeping $M$ capped. This requires us in turn to constrain $\alpha$ to small but finite values: a more systematic discussion of the procedure used to achieve this is given in Appendix II.

\section{Power transfer in a SIF with circular core and cladding}

We now outline how the power transport calculation works in the case of a SIF with circular core and cladding, using the specific geometry and initial conditions illustrated in Fig. 4 as a concrete example. Recall that there is no diffusion into whisperinggallery regions in this scenario, so we are free to take the limit $M \rightarrow \infty$ straightforwardly. However, because $T_{2 D}$ has many eigenvalues equal to unity (with eigenvectors supported in the whispering gallery regions of phase space and increasing in number as the pixelation is made finer), direct evaluation by (36) is not as simple as it was in the previous toy model.

We therefore evaluate $\Phi_{3 \mathrm{D}}$ by investigating the behavior of $\bar{\Phi}_{3 \mathrm{D}}(\alpha)$ for large $M$ and small $\alpha$, which is in any case is the approach we are compelled to use later for deformed SIFs. This behavior is presented in Fig. 9, providing an analogue of the calculations that were presented in Fig. 8 for the toy model. The greater computational complexity of this SIF example prevents us from pushing the calculations to iteration numbers as large as used in the previous toy model, but nevertheless the qualitative behavior is very similar. As $\alpha$ decreases, $\bar{\Phi}_{3 \mathrm{D}}(\alpha)$ saturates in Fig. 9 with increasing $M$ at limiting values that get ever closer to $\Phi_{3 \mathrm{D}}$. However, for the smallest values of $\alpha$ illustrated in Fig. 9, the maximum bounce number $M$ must be much larger than should be allowed for deformed SIFs if diffusion is not to dominate. We should therefore estimate $\Phi_{3 \mathrm{D}}$ using calculations confined to moderately small $\alpha$, the detailed procedure for which is set out in Appendix II.

\section{Power transfer in a SIF with deformed core and cladding}

Having established a procedure that enables us in problems without significant diffusion to estimate $\Phi_{3 \mathrm{D}}$, using moderate truncations in $M$, we now apply it to deformed SIFs, where diffusion is present. Plots of $\bar{\Phi}_{3 \mathrm{D}}(\alpha)$, as a function of $M$ are shown in Fig. 10 for several values of $\alpha$. This is the analogue of Figs. 8 and 9 for the fiber geometry and initial condition illustrated in Fig. 5.

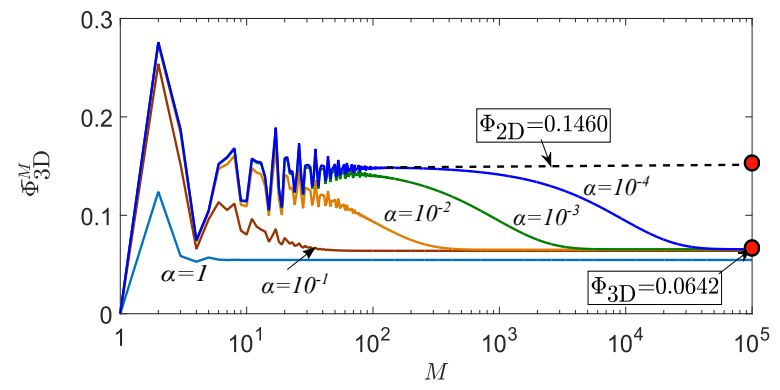

Fig. 9. The development of $\bar{\Phi}_{3 \mathrm{D}}^{M}(\alpha)$ with increasing $M$ is shown for a number of values of $\alpha$, similar to Fig. 8 but here for the SIF example with circular core-cladding boundaries. A corresponding plot with $\alpha=0$ here reproduces Fig. 6(a).

The main difference from previous calculations is that, because there is numerical diffusion, there is no clear intermediate plateau at a fixed value $\Phi_{2 \mathrm{D}}$ as $\alpha$ decreases. Instead, $\bar{\Phi}_{3 \mathrm{D}}(\alpha)$ continues to drift upwards as power diffuses out of the whispering gallery region of the cladding SOS and into the whispering gallery region of the core interior SOS. Nevertheless, the extrapolation procedure outlined in Appendix II allows us to obtain a physically meaningful estimate for $\Phi_{3 D}$. We fix $M$ at values large enough that there is convergence to an effective steady state (as determined from Fig. 6, for example), but small enough that diffusion does not dominate. We then extrapolate to $\alpha=0$ from values of $\alpha$ to that are moderately small, but not so small that $\bar{\Phi}_{3 \mathrm{D}}(\alpha)$ becomes sensitive to $M$. In the figure, the result is shown as an estimate $\Phi_{3 \mathrm{D}}=0.0672$ for the power flux transferred to the core from the initial cladding excitation.

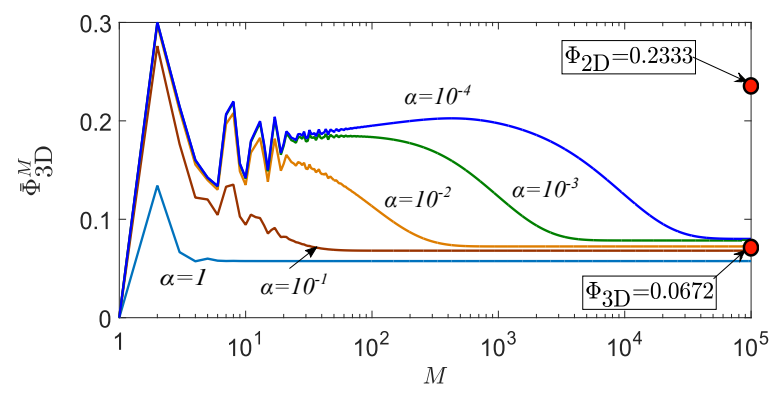

Fig. 10. An analog of Figs. 8 and 9 is shown here for the SIF example with deformed cladding and core. Numerical diffusion results in sustained drift that requires $\Phi_{3 \mathrm{D}}$ to be estimated while limiting $M$ to a few hundreds of reflections. A corresponding plot with $\alpha=0$ here reproduces Fig. 6(b).

\section{EXAMPLES WITH INPUT DISTRIBUTIONS}

We have established in Sec. 4 the proposed method for estimating power transfer to the core of a SIF, using simplified initial conditions. In this section we illustrate the application to a wider range of problems, describing in particular the influence of input distribution on the result. 
(a)

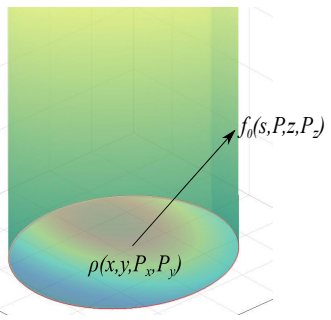

(b)

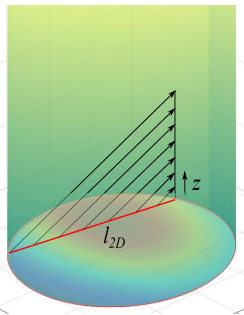

Fig. 11. In (a), the relation between first-arrival density $f_{0}\left(s, P, z, P_{z}\right)$ and input density $\rho\left(x, y, P_{x}, P_{y}\right)$ is illustrated. In (b), we illustrate the transformation from integration over $z$ to integration over a chord in the 2D projection, which is used in forming an initial condition in the Laplace domain.

\section{A. Initial conditions from input distributions}

The input for the calculation of power transfer to the core, as set out in Sec. 4 , is the density $f_{0}\left(s, P, z, P_{z}\right)$ of rays first arriving at the vertical boundary, before further reflection takes place. To complete the model we should relate this to the physically more accessible distribution of power injected at the input face $z=$ 0 : we denote this by $\rho\left(x, y, P_{x}, P_{y}\right)$, where Cartesian canonical coordinates $\left(x, y, P_{x}, P_{y}\right)$ are used to parameterize rays on the SOS defined by $z=0$. Using Liouville conservation of volume, these densities are simply related by

$$
f_{0}\left(s, P, z, P_{z}\right)=\rho\left(x, y, P_{x}, P_{y}\right),
$$

where coordinates $\left(x, y, P_{x}, P_{y}\right)$ provide the initial conditions on the input face for the ray arriving at the vertical boundary with coordinates $\left(s, P, z, P_{z}\right)$ (see Fig. 11(a)).

In fact, the calculation in Sec. 4 takes as input the partially integrated density

$$
\left.\bar{f}_{0}\left(s, P, \alpha, P_{z}\right)\right|_{\alpha=0}=\int_{0}^{\infty} f_{0}\left(s, P, z, P_{z}\right) \mathrm{d} z .
$$

In practice the integration over $z$ here is over a finite interval for given $\left(s, P, P_{z}\right)$, which can in turn be transformed to an integral over a chord in a $2 \mathrm{D}$ projection arriving at the projected coordinates, and using

$$
\mathrm{d} z=\frac{P_{z}}{\sqrt{n^{2}-P_{z}^{2}}} \mathrm{~d} l
$$

where $\mathrm{d} l$ denotes a length element over the projected chord (see Fig. 11(b).

\section{B. Variation of input distribution}

We now illustrate the effect of input distribution on the asymptotic power in the core using the examples of Gaussian distributions localized in different parts of the input face shown in Fig. 12. There are three cases, labelled G1, G2 and G3 respectively: G1 is localized in the core, G2 is localised in the cladding and G3 is also localised in the cladding, but further from the core and nearer the cladding boundary. These examples use the SIF-II geometry whose phase space is illustrated in Fig. 5, and the corresponding initial conditions $\bar{f}_{0}\left(s, P, \alpha=0, P_{z}\right)$ defined by them are shown to the right of Fig. 12, in each case for the value $P_{z}=0.75 n_{\mathrm{cl}}$.

The three examples are chosen to be localised initially in distinct parts of phase space. G1 defines an initial condition $\bar{f}_{0}\left(s, P, \alpha=0, P_{z}\right)$ that is concentrated in SOS(i). G2 and G3 both (a)
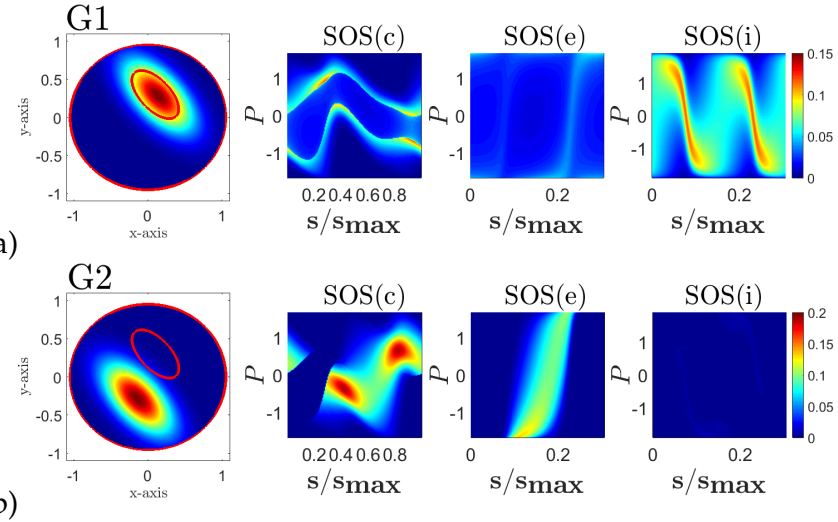

(b)
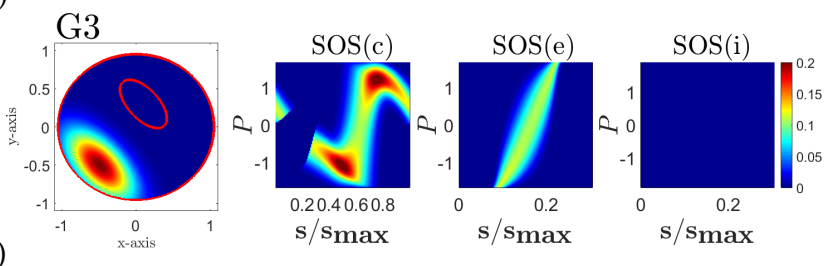

Fig. 12. Transfer of Gaussian distributions in the input face of the fiber G1, G2, and G3 to the curved boundary SOSs of SIF-II with $P_{z}=0.75 n_{\mathrm{cl}}$.

lead to $\bar{f}_{0}\left(s, P, \alpha=0, P_{z}\right)$ concentrated in SOS(e) and SOS(c), but with the case G3 showing greater overlap with the whispering gallery region of orbits trapped in the cladding phase space (red regions in the SOS(c) components of Fig. 5, whereas case G2 is initially localised primarily in the chaotic region (green regions in the SOS(c) components of Fig. 5).

For each of these examples we calculate the asymptotic power in the core following the method set out in Sec. 4, once for $P_{z}=0.75 n_{\mathrm{cl}}$ and then repeating the calculation for $P_{z}=0.98 n_{\mathrm{cl}}$, which is nearer the critical angle at which the core becomes completely guiding. The resulting plots of $\bar{\Phi}_{3 D}^{M}$ against $M$ are shown in Fig. 13, for a value of $\alpha=0.01$ that is small enough to show qualitatively the correct physical result (although the more detailed limiting procedure of Appendix II is used to find optimally accurate estimates).

We consider first the case $P_{z}=0.75 n_{\mathrm{cl}}$, shown by solid curves in Fig. 13. This is the value of $P_{z}$ previously used to illustrate phase space propagation in Fig. 5 and initial conditions $\bar{f}_{0}\left(s, P, \alpha=0, P_{z}\right)$ in Fig. 12. A striking aspect of these results is that the final outcomes of G1 and G2 are relatively close, while G3 shows an asymptotic power in the core that is somewhat lower than both. This is because even though G1 and G2 are respectively almost completely localized in the core and almost completely localized in the cladding, the core interior phase space is dominated by chaos and strongly coupled to the chaotic region of the cladding phase space. The asymptotic distribution for both initial conditions is then uniformly spread over this joint chaotic region. On the other hand G3 has a significant component that is initially localized in the whispering gallery region of the cladding, remaining forever inaccessible to the core and leading therefore to an asymptotic power in the core that is lower. Overall, because the core interior phase space is largely chaotic (see SOS(i) components of Fig. 5) it is strongly coupled to the cladding phase space and largely unguided as a result.

We also calculate net accumulation of power in the core for the case $P_{z}=0.98 n_{\mathrm{cl}}$, shown by dashed curves in Fig. 13. We 
have not shown explicit phase space plots for this value of $P_{z}$ but point out that the main differences to the previous case are that the whispering gallery region of the core is larger (the core is closer to being guiding) and the cladding phase space has an area that is a smaller fraction of the combined core and cladding phase space areas $\left(P_{1}\right.$ in Fig. 3 is relatively smaller than $P_{2}$ ). Compared to the previous calculations there is now a much greater difference between G1 and G2: the whispering gallery region of the core is relatively larger so the core is closer to being guiding and a significant fraction of the light starting in the core remains decoupled from the cladding. On the other hand, G2 and G3 show relatively closer asymptotic powers in the core: since the cladding phase space has a smaller overall weighting, the difference in the extent to which the whispering gallery region of the cladding is excited becomes less significant.

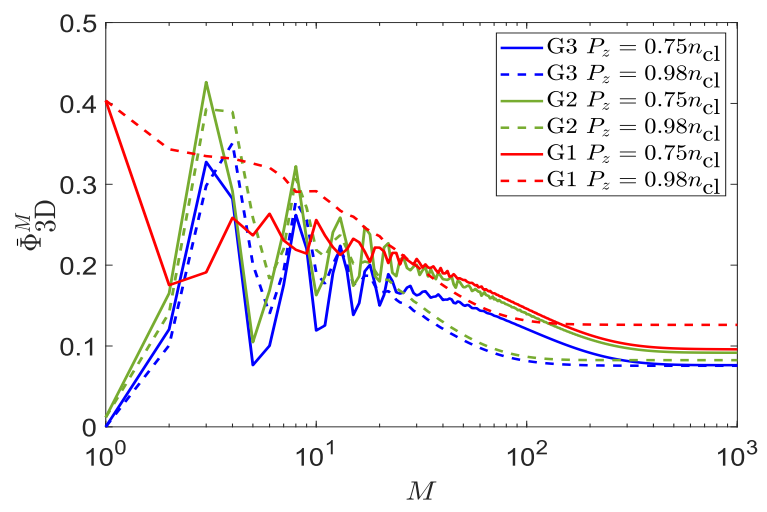

Fig. 13. The calculation of asymptotic power in the core is illustrated for input distributions G1, G2, and G3, for $P_{z}=$ $0.75 n_{\mathrm{cl}}$ (solid curves) and $P_{z}=0.98 n_{\mathrm{cl}}$ (dashed curves). These plots are analogs of Fig. 10 but for a fixed value of $\alpha=0.01$, chosen to be small enough that the asymptotic limit of the plots conveys qualitatively the required physical result.

\section{CONCLUSION}

A finite operator model has been used to model transport of power between core and cladding in optical SIFs. The model is based on a ray-tracing description of power transport and uses a coarse graining of phase space obtained by pixelating SOSs to reduce the problem to finite matrix calculations. By monitoring the transport of power flux across boundaries between core and cladding, the formalism can naturally be adapted to provide the net transfer of power between core and cladding following an initial excitation, which is of prime interest in applications.

The method can in particular be applied to deformed geometries with chaotic ray dynamics and complex borders between trapped and mixing regions of phase space. In the general case, however, diffusion artefacts brought about by the coarsegraining of phase space limit the accuracy of the simulation for a given pixel size. We have established a procedure to extract physically realistic estimates of power transfer in the face of these limitations and this has been benchmarked using simpler geometries that are not diffusion dominated.

We note that, although applied to lossless, gainless models in this paper, the underlying formalism can be adapted to treat light propagation in fibers with gain and/or losses, and it offers a numerically robust basis on which to design optical fibers for amplifier and fiber sensing applications.

\section{ACKNOWLEDGEMENTS}

DSK acknowledges, with thanks, the financial support of his PhD studies received from the Faculty of Engineering (Dean's Scholarship) and School of Mathematical Sciences, University of Nottingham, UK. TMB acknowledges, with thanks, the financial support of the Newton Fund International Links Award (ref. 277109657).

\section{APPENDIX I: PROPAGATION IN THE COARSE-GRAINED PHASE SPACE}

In this appendix we describe the basic mechanism that creates numerical diffusion in pixelated propagation of power densities. In concrete calculations we use the same example of an unstructured fiber as used in Sec. 3A

The SOS distribution created by iterating the finite operator and that obtained directly from ray-tracing are different after more than one bounce. Figure 14 illustrates the difference between them by showing the power arriving in target pixels from a single initial pixel after one (Figs. 14(a) and 14(b)) and two (Figs. 14(c) and 14(d)) reflections. As opposed to Fig. 2 this is for a (still rather coarse) $50 \times 50$ pixelation and for a $2 \mathrm{D}$ cross-section of an unstructured fiber described by (13). The ray-tracing version is obtained by multiply iterating the individual rays in the initial pixel and counting the fraction that ends up in each target pixel. For one iteration this simply repeats the calculation used to form the finite transfer operator and gives an identical result (Fig. 14(b)). The distributions obtained after two iterations, however, are different. In particular, the distribution obtained by iterating the finite transfer operator (Fig. 14(d)) reaches pixels that the directly ray-traced distribution (Fig. 14(c)) does not. This is a manifestation of diffusion brought about by coarse-graining the dynamics. (a)

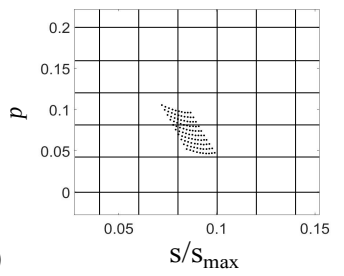

(c)

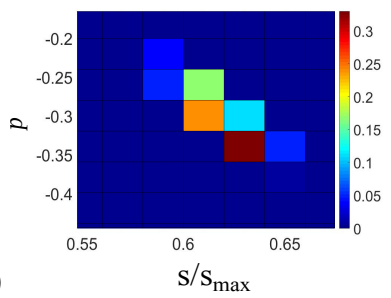

(b)

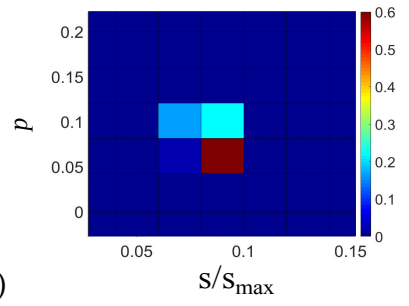

(d)

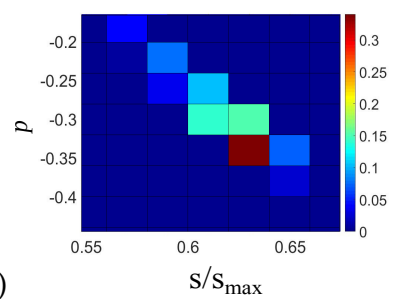

Fig. 14. (a) A set of rays from a pixel traced for the first bounce. (b) The corresponding intensity map of the ray distribution in a pixelated SOS with $N=50$. (c) Intensity map after raytracing twice (d) Intensity map after iterating with $\bar{T}_{2 \mathrm{D}}$ on the same initialization twice.

The directly ray-traced density is a more faithful representation of the true density but rapidly becomes infeasible as the 
number of iterations increases in problems with ray-splitting, such as a SIF, because of exponential proliferation of rays. The finite transfer matrix can easily be iterated, however, and provides a practical means of finding densities as long as we can mitigate the effects of diffusion artefacts.

To illustrate the significance of these artefacts we show in Fig. 15 a comparison between ray-traced and transfer-operatoriterated densities for a more realistic $300 \times 300$ pixelation of the same unstructured-fiber phase space. The deformed boundary in this example produces a mixed SOS, and an initial pixel is placed in the chaotic region of the SOS. The rays from the same pixel are traced for 150 reflections and the distribution obtained is compared with that obtained from the 2D finite operator. (Note that the ray tracing calculation is feasible here because there is no ray-splitting, but the corresponding calculation in a SIF would not be.) In the ray-traced version shown in Fig. 15(a), the separation between the chaotic sea and the regular islands has been maintained. By contrast, the density produced by the finite operator method. shown in Fig. 15(b), leaks into the regions occupied by regular islands of the true dynamics, as highlighted by the circles. These diffusion artefacts can be decreased by using finer pixelations but can never be eliminated in practice so we must in our final calculations constrain iteration numbers so that such artefacts are not dominant. (a)

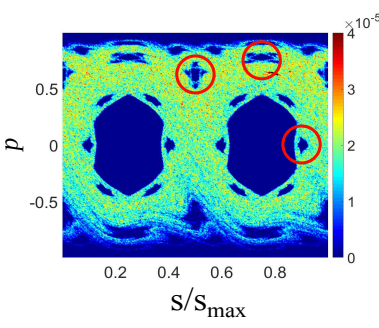

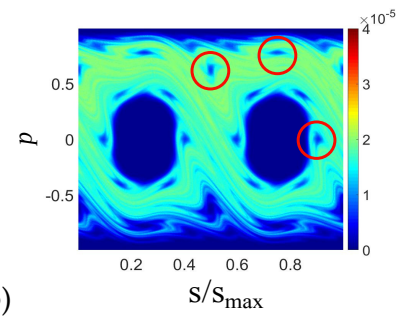

(b) (a)
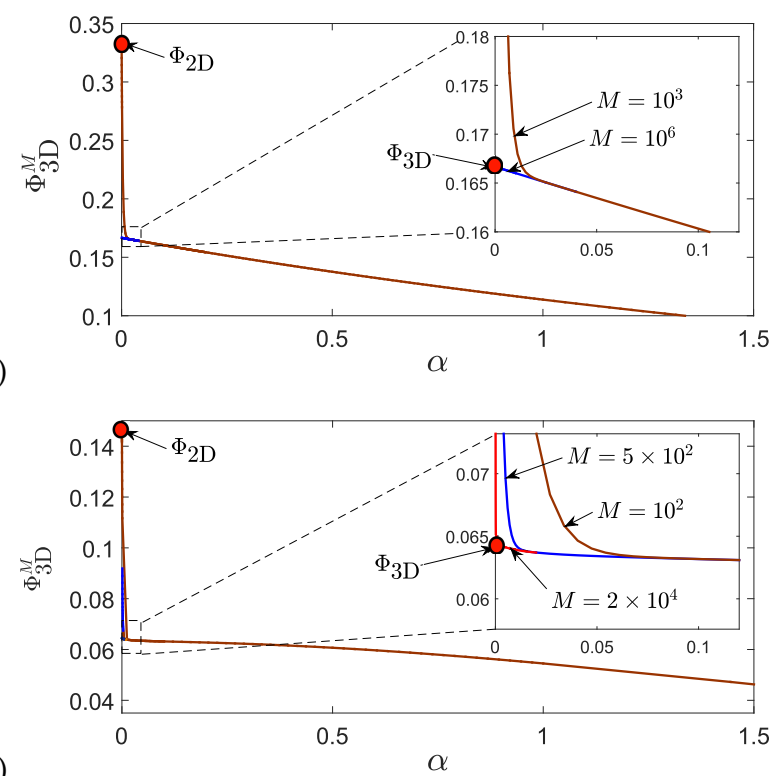

(b)

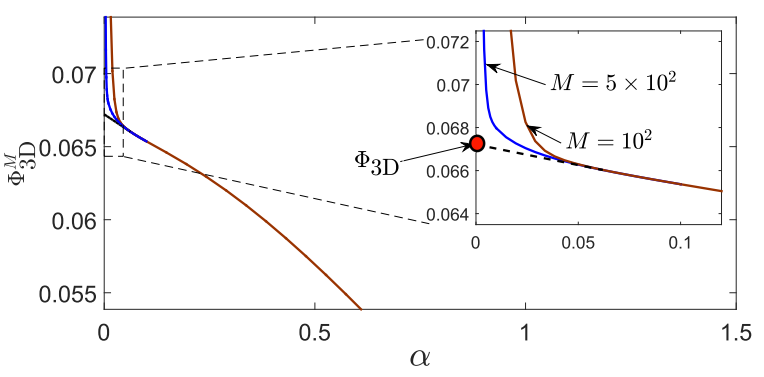

(c)

Fig. 16. For each of the three example structures discussed in Sec. 4 , we show the variation of $\bar{\Phi}_{3 \mathrm{D}}(\alpha)$ with $\alpha$ for selected values of $M$. In the last two examples these values of $M$ are chosen in the range where numerical diffusion would not yet dominate the flux transfer calculation.

iterated intensity map in a pixelated $(N=300)$, and mixed SOS. A single pixel in the chaotic region was initialized and propagated for 150 bounces around the deformed boundary. The separation between the regular regions and the chaotic sea is maintained in the ray-traced SOS but in the $\bar{T}_{2 \mathrm{D}}$ iterated SOS the intensities in the two regions can overlap, as highlighted by the circles.

\section{APPENDIX II: ESTIMATES OF POWER TRANSFER US- ING FINITE NUMBERS OF BOUNCES}

In each of the three example structures used in Sec. 4 to illustrate the calculation of flux transfer to the core (the toy model, the SIF with circular core and cladding and the SIF with deformed core and cladding), we provided estimates of $\Phi_{3 \mathrm{D}}$ obtained while restricting the maximum number of bounces $M$ to moderately large values, constrained so that numerical diffusion should not dominate. In this appendix we provide details of the quantitative procedure used to achieve such estimates.

We begin with the toy model, for which complete, effectively analytical results are available for all quantities of interest and there are no diffusion artefacts. In Fig. 16(a) we plot $\bar{\Phi}_{3 \mathrm{D}}(\alpha)$ for this toy model as a function of $\alpha$ for selected values of $M$. As $\alpha$ decreases, $\bar{\Phi}_{3 \mathrm{D}}^{M}(\alpha)$ appears to converge to a fixed value approximating $\Phi_{3 \mathrm{D}}$ until a layer of very small values of $\alpha$ is reached in which $\bar{\Phi}_{3 \mathrm{D}}^{M}(\alpha)$ increases sharply towards $\Phi_{2 \mathrm{D}}$. Before this layer is reached (as $\alpha$ decreases), the value of $M$ is large enough that the geometric sum implicit in (32) has converged to its limiting value and the result is insensitive to $M$ - extrapolating from this exterior region allows us to approximate $\Phi_{3 \mathrm{D}}$ effectively (see insert).

In the toy model, this extrapolation procedure simply reproduces the result that can be obtained by passing directly to the limit $M \rightarrow \infty$ as in (36). For 3D SIFs, however, direct evaluation is harder, or not appropriate, so we are restricted to the extapolation procedure. The corresponding plots are shown in Figs. 16(b) and 16(c), respectively for the examples with circular and deformed core and cladding. In these examples we restrict $M$ to values in the hundreds so that diffusion artefacts are not important and $\bar{\Phi}_{3 \mathrm{D}}$ is similarly estimated by extrapolating to $\alpha=0$ from small values of $\alpha$ but excluding the layer of extremely small values in which the geometric sum implicit in (32) is sensitive to the finite value of $M$. Note that the plots and extrapolation are similar in both of these cases, despite the qualitative differences between Figs. 9 and 10 arising from diffusive drift (or lack of it).

\section{REFERENCES}

1. A. B. Seddon, "A prospective for new mid-infrared medical endoscopy using chalcogenide glasses," Int. J. Appl. Glass Sci. 2, 177-191 (2011). 
2. F. Starecki, F. Charpentier, J.-L. Doualan, L. Quetel, K. Michel, R. Chahal, J. Troles, B. Bureau, A. Braud, P. Camy, V. Moizan, and V. Nazabal, "Mid-IR optical sensor for $\mathrm{CO}_{2}$ detection based on fluorescence absorbance of $\mathrm{Dy}^{3+}$ : $\mathrm{Ga}_{5} \mathrm{Ge}_{20} \mathrm{Sb}_{10} \mathrm{~S}_{65}$ fiber," Sensors Actuators B: Chem. 207, $518-525$ (2015).

3. R. Chahal, F. Starecki, C. Boussard-Plédel, J.-L. Doualan, K. Michel, L. Brilland, A. Braud, P. Camy, B. Bureau, and V. Nazabal, "Fiber evanescent wave spectroscopy based on IR fluorescent chalcogenide fibers," Sensors Actuators B: Chem. 229, 209 - 216 (2016).

4. Z. Tang, V. S. Shiryaev, D. Furniss, L. Sojka, S. Sujecki, T. M. Benson, A. B. Seddon, and M. F. Churbanov, "Low loss Ge-As-Se chalcogenide glass fiber, fabricated using extruded preform, for mid-infrared photonics," Opt. Mater. Express 5, 1722-1737 (2015).

5. V. Shiryaev and M. Churbanov, "Trends and prospects for development of chalcogenide fibers for mid-infrared transmission," J. Non-Crystalline Solids 377, $225-230$ (2013). ISNOG 2012 Proceedings of the 18th International Symposium on Non-Oxide and New Optical Glasses Rennes, France, July 1-5, 2012.

6. F. Starecki, N. Abdellaoui, A. Braud, J.-L. Doualan, C. Boussard-Plédel, B. Bureau, P. Camy, and V. Nazabal, " $8 \mu \mathrm{m}$ luminescence from a Tb ${ }^{3+}$ GaGeSbSe fiber," Opt. Lett. 43, 1211-1214 (2018).

7. L. Sojka, Z. Tang, D. Furniss, H. Sakr, Y. Fang, E. Beres-Pawlik, T. M. Benson, A. B. Seddon, and S. Sujecki, "Mid-infrared emission in $\mathrm{Tb}^{3+}$. doped selenide glass fiber," J. Opt. Soc. Am. B 34, A70-A79 (2017).

8. H. Parnell, D. Furniss, Z. Tang, N. C. Neate, T. M. Benson, and A. B. Seddon, "Compositional dependence of crystallization in $\mathrm{Ge}-\mathrm{Sb}-\mathrm{Se}$ glasses relevant to optical fiber making," J. Am. Ceram. Soc. 101, 208-219 (2018).

9. S. D. Savage, C. A. Miller, D. Furniss, and A. B. Seddon, "Extrusion of chalcogenide glass preforms and drawing to multimode optical fibers," J. Non-Crystalline Solids 354, $3418-3427$ (2008).

10. Z. Tang, D. Furniss, M. Fay, H. Sakr, L. Sójka, N. Neate, N. Weston, S. Sujecki, T. M. Benson, and A. B. Seddon, "Mid-infrared photoluminescence in small-core fiber of praseodymium-ion doped selenide-based chalcogenide glass," Opt. Mater. Express 5, 870-886 (2015).

11. D. Jayasuriya, "Towards mid-infrared fiber-optic devices and systems for sensing, mapping and imaging," in "PhD thesis, University of Nottingham," (2018).

12. V. Doya, O. Legrand, and F. Mortessagne, "Optimized absorption in a chaotic double-clad fiber amplifier." Opt. letters 26, 872-874 (2001).

13. P. Leproux, S. Février, V. Doya, P. Roy, and D. Pagnoux, "Modeling and Optimization of Double-Clad Fiber Amplifiers Using Chaotic Propagation of the Pump," Opt. Fiber Technol. 7, 324-339 (2001).

14. L. Philippe, V. Doya, R. Philippe, P. Dominique, M. Fabrice, and L. Olivier, "Experimental study of pump power absorption along rareearth-doped double clad optical fibres," Opt. Commun. 218, 249-254 (2003).

15. H.-J. Stöckmann and J. Stein, "Quantum chaos in billiards studied by microwave absorption," Phys. Rev. Lett. 64, 2215-2218 (1990).

16. M. J. Giannoni, A. Voros, and J. Zinn-Justin, "Chaos and quantum physics," (Proc. Les Houches, North-Holland, Amsterdam, 1989).

17. J. U. Nöckel, A. D. Stone, and R. K. Chang, "Q spoiling and directionality in deformed ring cavities," Opt. Lett. 19, 1693-1695 (1994).

18. A. Mekis, J. U. Nöckel, G. Chen, A. D. Stone, and R. K. Chang, "Ray chaos and q spoiling in lasing droplets," Phys. Rev. Lett. 75, 2682-2685 (1995).

19. J. Wiersig, "Formation of long-lived, scarlike modes near avoided resonance crossings in optical microcavities," Phys. Rev. Lett. 97, 253901 (2006).

20. C. Michel, V. Doya, O. Legrand, and F. Mortessagne, "Selective amplification of scars in a chaotic optical fiber," Phys. Rev. Lett. 99, 1-4 (2007).

21. C. Michel, S. Tascu, V. Doya, O. Legrand, and F. Mortessagne, "Gaincontrolled wave chaos in a chaotic optical fiber," J. Eur. Opt. Soc. Rapid publications 4, 09020 (2009).

22. C. Michel, M. Allgaier, and V. Doya, "Regular modes in a mixeddynamics-based optical fiber," Phys. Rev. E 93, 022201 (2016).

23. M. Tabor, Chaos and integrability in nonlinear dynamics : an introduc- tion (Wiley, 1951).

24. E. Ott, Chaos in Dynamical Systems (New York: Cambridge University Press, 1993)

25. K. M. Frahm and D. L. Shepelyansky, "Ulam method for the Chirikov standard map," Eur. Phys. J. B 76, 57-68 (2010).

26. G. Froyland, "Ulam's method for random interval maps," Nonlinearity 12, 1029-1051 (1999).

27. L. Ermann and D. L. Shepelyansky, "The Arnold cat map, the Ulam method and time reversal," Phys. D: Nonlinear Phenom. 241, 514-518 (2012).

28. J. Kullig and J. Wiersig, "Frobenius-Perron eigenstates in deformed microdisk cavities: Non-Hermitian physics and asymmetric backscattering in ray dynamics," New J. Phys. 18, 15005 (2016).

29. J. Wiersig, "Chiral and nonorthogonal eigenstate pairs in open quantum systems with weak backscattering between counterpropagating traveling waves," Phys. Rev. A - At. Mol. Opt. Phys. 89, 1-7 (2014). 